This item was submitted to Loughborough's Research Repository by the author.

Items in Figshare are protected by copyright, with all rights reserved, unless otherwise indicated.

\title{
Recognition of proximally phosphorylated tyrosine residues and continuous analysis of phosphatase activity using a stable europium complex
}

\section{PLEASE CITE THE PUBLISHED VERSION}

https://doi.org/10.1080/10610278.2017.1410548

\section{PUBLISHER}

(c) Taylor and Francis

\section{VERSION}

AM (Accepted Manuscript)

\section{PUBLISHER STATEMENT}

This work is made available according to the conditions of the Creative Commons Attribution-NonCommercialNoDerivatives 4.0 International (CC BY-NC-ND 4.0) licence. Full details of this licence are available at: https://creativecommons.org/licenses/by-nc-nd/4.0/

\section{LICENCE}

CC BY-NC-ND 4.0

\section{REPOSITORY RECORD}

Hewitt, Sarah, Roanna Liu, and Stephen Butler. 2017. "Recognition of Proximally Phosphorylated Tyrosine Residues and Continuous Analysis of Phosphatase Activity Using a Stable Europium Complex". figshare. https://hdl.handle.net/2134/28343. 
Recognition of proximally phosphorylated tyrosine residues and continuous analysis of phosphatase activity using a stable europium complex

Sarah H. Hewitt ${ }^{\mathrm{a}}$, Roanna Liu ${ }^{\mathrm{a}}$ and Stephen J. Butler ${ }^{\mathrm{a} *}$

${ }^{a}$ Department of Chemistry, Loughborough University, Loughborough, LE11 3TU, UK.

Email: s.j.butler@lboro.ac.uk; Tel: +44 (0)1509 222577 


\title{
Recognition of proximally phosphorylated tyrosine residues and continuous analysis of phosphatase activity using a stable europium complex
}

\begin{abstract}
The recognition of proteins and their post-translational modifications using synthetic molecules is an active area of research. A common post-translational modification is the phosphorylation of serine, threonine or tyrosine residues. The phosphorylation of proximal tyrosine residues occurs in over 1000 proteins in the human proteome, including in disease-related proteins, so the recognition of this motif is of particular interest. We have developed a luminescent europium(III) complex, $[\mathbf{E u . 1}]^{+}$, capable of the discrimination of proximally phosphorylated tyrosine residues, from analogous mono- and non-phosphorylated tyrosine residues, more distantly-related phosphotyrosine residues and over proximally phosphorylated serine and threonine residues. $[\mathbf{E u . 1}]^{+}$was used to continuously monitor the phosphatase catalysed dephosphorylation of a peptide containing proximally phosphorylated tyrosine residues.
\end{abstract}

Keywords: anion sensing; luminescence; europium; phosphorylation; enzyme assay

\section{Introduction}

The detection and discrimination of proteins and their post-translational modifications using synthetic molecules is an active area of research. ${ }^{1-4}$ Traditional approaches for the discrimination of proteins involve the use of enzyme and antibody responses (for example using ELISA), ${ }^{5}$ which can be cumbersome and expensive. Synthetic molecules may offer advantages in terms of stability, their tunable luminescence properties and their potentially cheaper preparation. One posttranslational modification of particular importance is the phosphorylation of serine, threonine and tyrosine residues, which acts as key regulators of protein function and signalling within biological systems. ${ }^{6}$ The study of the phosphorylation and dephosphorylation of proteins, catalysed by kinases and phosphatases respectively, is of 
critical importance both for the understanding of biological processes and facilitating drug discovery.

The phosphorylation of tyrosine residues is less common $(\sim 1 \%)$ compared to that of serine $(\sim 90 \%)$ or threonine $(\sim 4 \%)$ residues. However, the phosphorylation of proximal tyrosine residues occurs in over 1000 proteins in the human proteome, ${ }^{7}$ including in disease-related proteins such as JAK2 and IGFR proteins. Therefore, the development of synthetic probes for the detection of proximal phosphotyrosine (pY) residues within peptides and proteins, with minimal interference from phosphoserine $(\mathrm{pS})$ or phosphothreonine $(\mathrm{pT})$ residues, could be useful for more specific sensing of these proteins. Additionally, such probes could be used for screening of new drugs for the treatment of diseases caused by abnormal phosphorylation.

The detection of phosphorylated amino acid residues within peptides and proteins has been achieved using discrete emissive metal complexes, including $\mathrm{Zn}(\mathrm{II}){ }^{8-}$ ${ }^{11} \mathrm{Cu}(\mathrm{II}),{ }^{12} \mathrm{~Tb}(\mathrm{III}),{ }^{13-14}$ and $\mathrm{Eu}(\mathrm{III})$ complexes, ${ }^{15-16}$ whereby coordination of the phosphorylated residue to the metal complex induces a measurable change in luminescence. However, these probes often show very little sequence specificity or selectivity between phosphorylated amino acid residues. Some sensing selectivity has been achieved for certain phosphorylation patterns, including proximally phosphorylated tyrosine residues. The Hamachi group have worked extensively with $\mathrm{Zn}$ (II)-dipicolyamine complexes for the detection of phosphorylated peptides and have utilised selected probes for both end-point phosphatase assays and real-time monitoring. ${ }^{8,10,17}$ Gunning and coworkers prepared a fluorescent Zn(II)-cyclen complex for the detection of proximal di- and triphosphorylated proteins, ${ }^{11}$ demonstrating its ability to discriminate proteins on a polyacrylamide gel. However, this probe cannot discriminate proximal $\mathrm{pY}$ residues ( $\mathrm{pYpY}$ motifs) from peptides 
containing a $\mathrm{pYXpY}$ motif (where $\mathrm{X}$ is any amino acid) nor a $\mathrm{pYpT}$ motif, ${ }^{18}$ with similar spectral responses reported. Increased specificity for a target phosphorylated peptide has been achieved by conjugation of various emissive metal complexes to a designed peptide or protein; ${ }^{19-25}$ however, this approach involves additional non-trivial synthetic processes.

Herein we report the use of a stable europium(III) complex, $[\text { Eu.1 }]^{+}$(Figure 1a), for the detection of proximally phosphorylated tyrosine residues within a peptide, and demonstrate the probe's utility in monitoring an acid phosphatase catalysed dephosphorylation reaction in real-time. The use of a discrete luminescent $\mathrm{Eu}(\mathrm{III})$ complex for phosphopeptide recognition and monitoring enzyme activity offers several advantages, including line-like emission spectra that permits ratiometric detection, large pseudo Stokes' shifts and the potential for time-resolved emission spectra, permitting complete removal of background autofluorescence arising from peptide or protein substrates. $^{25-32}$
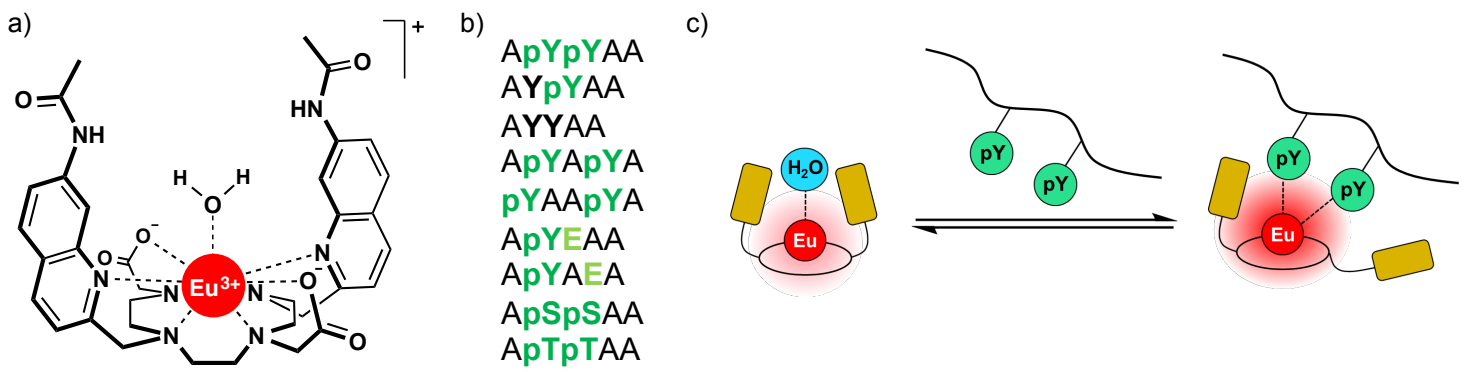

[Eu.1 $]^{+}$

Figure 1. a) Structure of complex [Eu.1 $]^{+}$; b) Different peptides used in this study; c) Cartoon representation of the proposed binding mode of $[\mathbf{E u . 1}]^{+}$to peptides containing the pYpY motif.

We have previously reported the synthesis ${ }^{33}$ and application of $[\mathbf{E u . 1}]^{+}$for the detection of nucleotide polyphosphate anions, ${ }^{34}$ using the probe to monitor the ratio of ADP/ATP during the kinase catalysed phosphorylation of a serine containing peptide. 
We now report another application for this Eu(III) complex: the luminescent detection of proximally phosphorylated tyrosine (pYpY) residues within peptides. This spectral response of [Eu.1 $]^{+}$is distinct compared to mono- and non-phosphorylated tyrosine residues (YpY and YY), proximally phosphorylated serine (pSpS) and threonine (pTpT) residues and more distant phosphorylated tyrosine residues (e.g. pYApY and pYAApY). The excellent discriminatory behaviour of [Eu.1 $]^{+}$allowed the phosphatase catalysed dephosphorylation of a proximally phosphorylated peptide, ApYpYAA, to be monitored in real-time.

\section{Results and Discussion}

\section{Discrimination of proximally phosphorylated tyrosine residues}
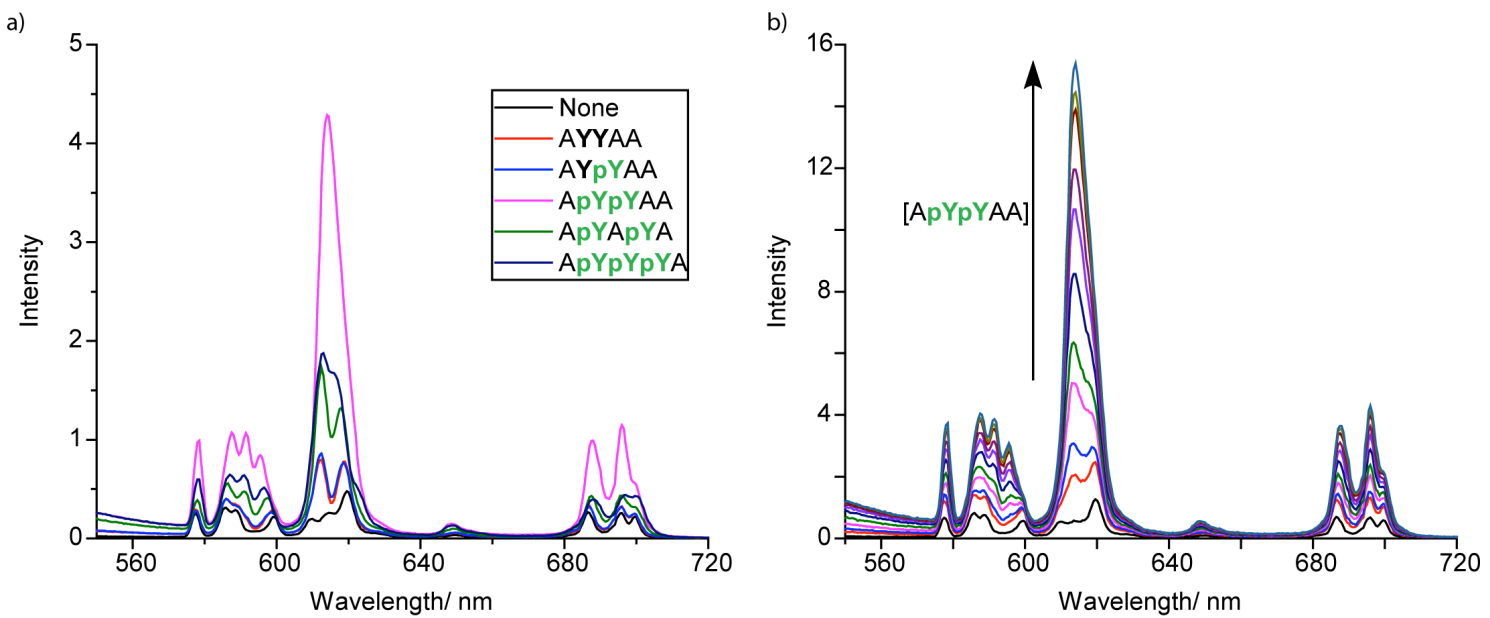

Figure 2. a) Luminescence response of $[\mathbf{E u} .1]^{+}(8 \mu \mathrm{M})$ in the presence of different phosphotyrosine containing peptides $(10 \mathrm{mM})$; b) Titration of ApYpYAA $(0-8 \mathrm{mM})$ into [Eu.1 $]^{+}(8 \mu \mathrm{M})$. Conditions: $10 \mathrm{mM}$ HEPES, $\mathrm{pH} 7.0, \lambda_{\mathrm{exc}}=330 \mathrm{~nm}$

Figure 2a shows the change in emission intensity of [Eu.1 $]^{+}$in the presence of various phosphotyrosine containing peptides $(10 \mathrm{mM})$, measured in aqueous buffer $(10$ mM HEPES) at $\mathrm{pH}$ 7.0. Addition of the proximally phosphorylated peptide, ApYpYAA, resulted in a striking increase in $\mathrm{Eu}(\mathrm{III})$ emission intensity and a 
pronounced change in spectral form. The emission spectral response was much lower for the other peptides tested, including the analogous mono- and non-phosphorylated peptides, AYpYAA and AYYAA, a proximally triphosphorylated peptide ApYpYpYA, and a diphosphorylated peptide bearing two $\mathrm{pY}$ residues separated by a single alanine residue (ApYApYA). The distinctive response to the $\mathrm{pYpY}$ containing peptide lead us to explore the use of $[\mathbf{E u . 1}]^{+}$for the recognition of the pYpY motif over a wider range of different phosphorylated peptides.
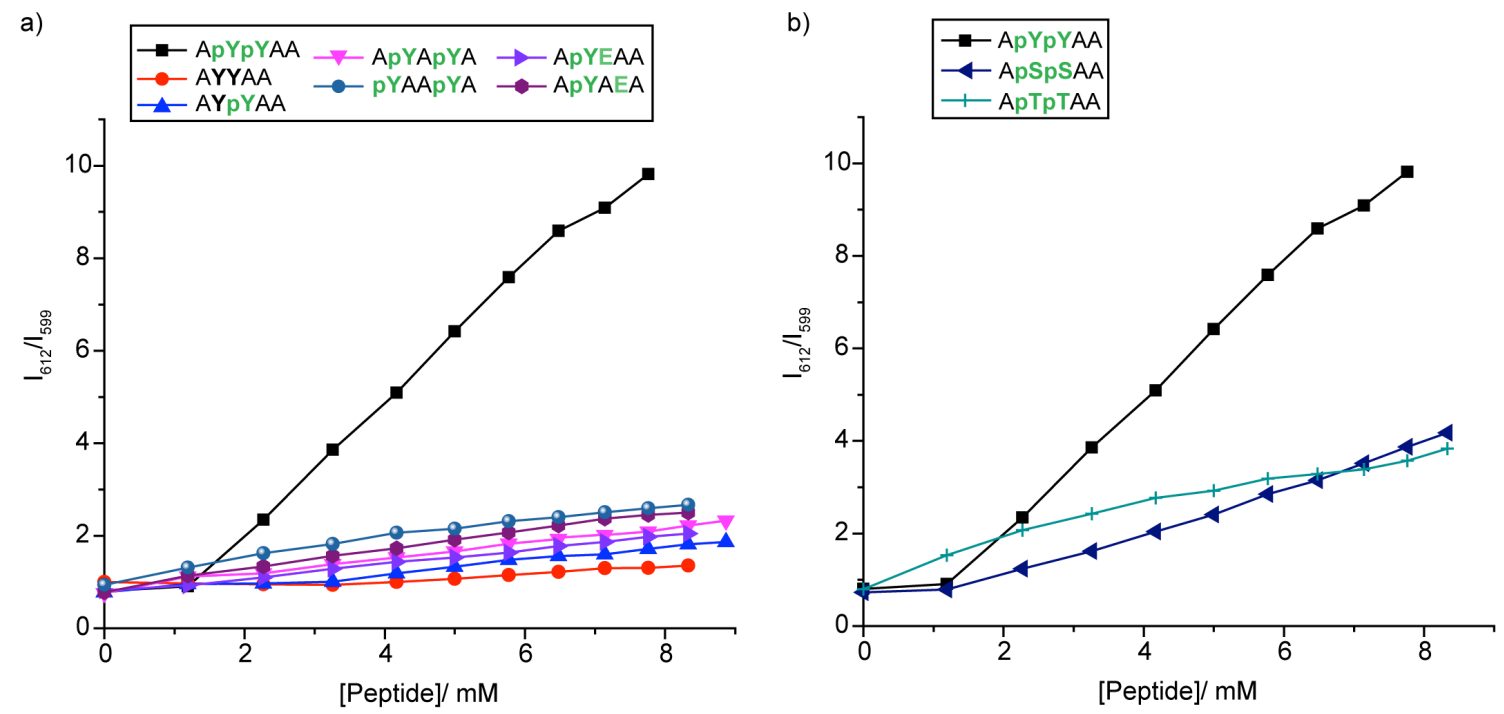

Figure 3. Change in the emission intensity ratio of [Eu.1 $]^{+}(8 \mu \mathrm{M})$ at $612 / 599 \mathrm{~nm}$ upon incremental addition of: a) various phosphotyrosine ( $\mathrm{pY}$ ) containing peptides; $b$ ) proximally diphosphorylated tyrosine, serine and threonine containing peptides. Conditions: $10 \mathrm{mM}$ HEPES buffer, $\mathrm{pH} 7.0, \lambda_{\mathrm{exc}}=330 \mathrm{~nm}$.

A titration of the proximally phosphorylated tyrosine peptide, ApYpYAA, into [Eu.1] ${ }^{+}$caused a rapid increase in intensity of the $\Delta J=2$ emission band centred around $614 \mathrm{~nm}$ (Figure 2b). In sharp contrast, titrations of a range of other phosphopeptides resulted in much smaller changes in $\mathrm{Eu}(\mathrm{III})$ emission intensity (Figure S1). The high sensing selectivity for the peptide ApYpYAA can be visualised clearly by following the ratiometric change in intensity at $612 / 599 \mathrm{~nm}$ as a function of peptide concentration 
(Figure 3a). Addition of ApYpYAA (0-8 mM) caused a 10-fold enhancement in the emission intensity ratio, whereas the mono- and non-phosphorylated peptides (AYpYAA and AYYAA, respectively) induced less than 10\% increases in the ratiometric signal. Similarly, only minor increases in the emission intensity were observed upon addition of peptides containing two more distantly related phosphotyrosine residues $(i, i+2$ for ApYApYA, or $i, i+3$ for pYAApYA). This is significant, as previously reported synthetic probes exhibited limited selectivity between peptides containing differentially located phosphorylated residues. ${ }^{18,35-37}$ Importantly, replacing one of the $\mathrm{pY}$ residues with a negatively charged glutamic acid residue (ApYEAA or ApYAEA, both purple in Figure 3a), resulted in minimal increases in the intensity ratio at $612 / 599 \mathrm{~nm}$ upon titration of these peptides. These results demonstrate that the presence of a pYpY motif is essential for the distinctive response of [Eu.1 $]^{+}$, rather than there simply being a requirement for two adjacent negatively charged amino acid residues.

Table 1. Luminescence lifetimes in $\mathrm{H}_{2} \mathrm{O}$ and $\mathrm{D}_{2} \mathrm{O}$ (10 mM HEPES, $\mathrm{pH}$ 7.0) and calculated $q$ values for $[\mathbf{E u . 1}]^{+}(30 \mu \mathrm{M})$ alone and in the presence of AYYAA, AYpYAA and ApYpYAA (all $9 \mathrm{mM}$ ); $\lambda_{\mathrm{exc}}=330 \mathrm{~nm}, \lambda_{\mathrm{em}}=615 \mathrm{~nm}$.

\begin{tabular}{|c|c|c|c|}
\hline peptide & $\boldsymbol{\tau}_{\mathbf{H}_{2} \mathbf{O}} / \mathbf{m s}$ & $\boldsymbol{\tau}_{\mathbf{D}_{2} \mathbf{O}} / \mathbf{m s}$ & $\boldsymbol{q}^{\mathbf{a}}$ \\
\hline none & 0.55 & 1.22 & 0.8 \\
AYYAA & 0.68 & 1.30 & 0.5 \\
AYpYAA & 0.72 & 1.21 & 0.3 \\
ApYpYAA & 0.89 & 1.27 & 0.1 \\
\hline
\end{tabular}

${ }^{a}$ Values of hydration state, $q(20 \%)$ are derived using methods in ref. 38. 
The discrimination of the pYpY motif is likely to arise from both higher affinity binding and a distinctive binding mode to the $\mathrm{Eu}(\mathrm{III})$ complex. When the two proximal pY residues are present, it is hypothesised that both phosphate groups coordinate to the $\mathrm{Eu}(\mathrm{III})$ metal, causing displacement of the bound water molecule and potentially one of the coordinated quinoline arms, allowing multi-dentate binding (Figure 1c). Evidence for a change in coordination environment of the $\mathrm{Eu}(\mathrm{III})$ metal is given by a change in the structure of the hypersensitive $\Delta J=2$ band and an increase in the emission lifetime of $[\mathbf{E u . 1}]^{+}$in the presence of ApYpYAA compared to the Eu(III) complex alone (Table 1). Calculation of hydration state, $q$, indicates displacement of the bound water molecule from [Eu.1 $]^{+}$on incubation with the ApYpYAA peptide (Table 1). ${ }^{38}$ A smaller increase in the emission lifetime of $[\mathbf{E u . 1}]^{+}$in the presence of the AYYAA and the AYpYAA peptide compared to the $\mathrm{Eu}(\mathrm{III})$ complex alone, is also observed, with the non-integral $q$ values indicating formation of 'partially' hydrated species on incubation with these peptides. These peptides also induce small changes in the spectral form of the $\Delta J=2$ band of [Eu.1 $]^{+}$, but minimal changes in the $\Delta J=1$ band, compared to pronounced changes in both bands on incubation with ApYpYAA (Figure S2). This indicates a change in ligand field on incubation with all three peptides, however the different spectral changes, along with the larger change in $q$ in the presence of ApYpYAA indicate a different binding mode. Furthermore, it is reasonable to suggest that this binding mode may only be possible when two proximal pY residues are present in the peptide, as further spacing between $\mathrm{pY}$ residues results in less favourable entropy of binding, as seen by the minimal changes in spectral form upon addition ApYApYA and ApYAApY.

Next, we examined the ability of $[\mathbf{E u . 1}]^{+}$to distinguish between peptides bearing proximal $\mathrm{pY}, \mathrm{pS}$ and $\mathrm{pT}$ residues. Titrations of peptides bearing proximally 
phosphorylated serine and threonine residues (ApSpSAA and ApTpTAA) caused approximately 4-fold enhancements in the $612 / 599 \mathrm{~nm}$ emission intensity ratio of $[\mathbf{E u . 1}]^{+}$, significantly lower than the analogous diphosphotyrosine peptide, ApYpYAA (Figure $3 \mathrm{~b}$ and Figure S3). Thus, $[\text { Eu.1 }]^{+}$can be used to detect peptides containing proximal $\mathrm{pY}$ residues over proximal $\mathrm{pS}$ or $\mathrm{pT}$ residues. This could be attributed to a change in the orientation of the phosphate groups compared to the peptide backbone and the potential for $\pi-\pi$ stacking between the coordinated $\mathrm{pY}$ residues and the quinoline arms of the $\mathrm{Eu}(\mathrm{III})$ complex. Differences in the binding orientation between the diphosphorylated peptides is indicated by the clear differences in fine splitting of the $\Delta J$ $=1$ emission band (Figure S4). ${ }^{39}$ Additionally, the lower desolvation energy of $\mathrm{pY}$ residues compared to $\mathrm{pS}$ or $\mathrm{pT}$ residues is expected to contribute towards the overall free energy of binding of pYpY containing peptides to [Eu.1 $]^{+}$.

\section{Continuous monitoring of a phosphatase enzyme reaction}

Having shown that $[\mathbf{E u . 1}]^{+}$can distinguish a peptide containing proximal pY residues from other non-phosphorylated, monophosphorylated and diphosphorylated peptides, we wished to utilise the probe to monitor a phosphatase-catalysed dephosphorylation reaction. For this proof-of-concept enzyme reaction we used acid phosphatase, an enzyme which catalyses the hydrolysis of phosphate monoesters, including from $\mathrm{pY}, \mathrm{pS}$ and $\mathrm{pT}$ residues, within the $\mathrm{pH}$ range $4.0-7.0$. The enzyme is therefore expected to remove both the phosphate groups from the ApYpYAA peptide, forming AYYAA via either ApYYAA or AYpYAA. 
a)

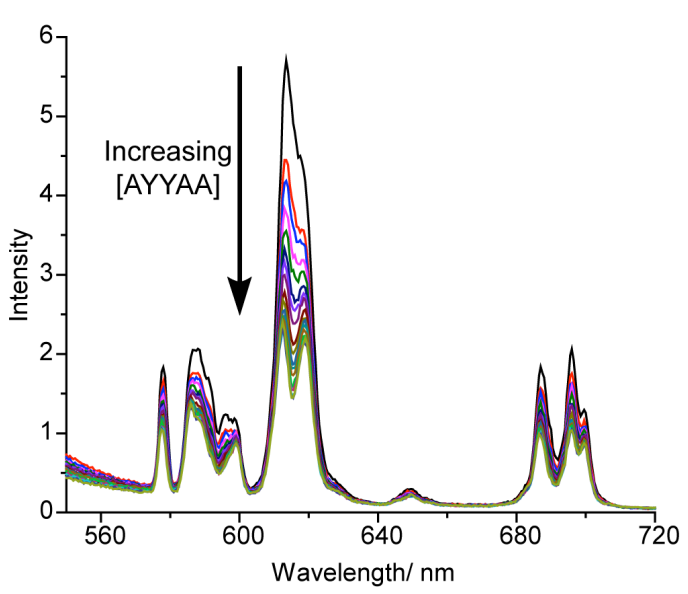

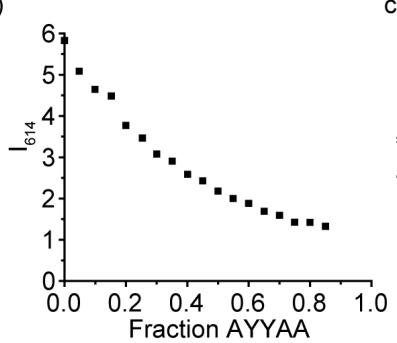

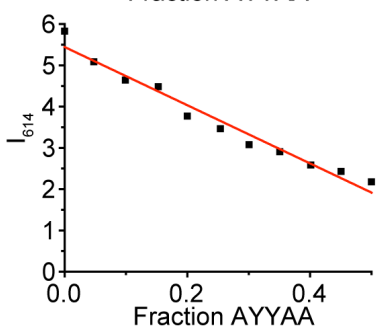

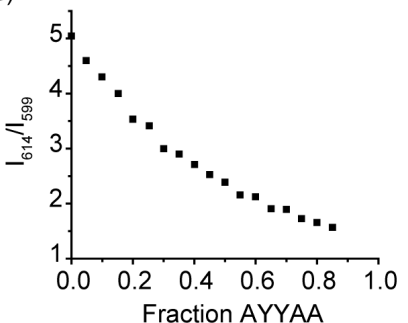

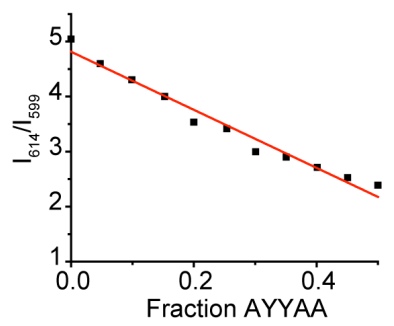

Figure 4. Model of the phosphatase reaction, by titrating peptide AYYAA $(0-3.4 \mathrm{mM})$ into a solution containing ApYpYAA (4 mM initially) and [Eu.1 $]^{+}(8 \mu \mathrm{M})$, monitoring the change in emission intensity. a) Change in emission spectra of $[\mathbf{E u . 1}]^{+}$; b) Change in the emission intensity at $614 \mathrm{~nm}$ over the whole titration (upper) and in the linear range (lower); c) Change in the intensity ratio at 614/599 nm over the whole titration (upper) and in the linear range (lower). Conditions: $10 \mathrm{mM}$ HEPES buffer, $\mathrm{pH} 7.0, \lambda_{\mathrm{exc}}=330$ nm.

Before pursuing the enzyme reaction, we modelled the change in $\mathrm{Eu}(\mathrm{III})$ emission spectra that might be expected during the enzymatic conversion of peptide ApYpYAA to both the mono- and non-phosphorylated analogues, AYpYAA and AYYAA (Figure S5 and Figure 4, respectively). Titrations of AYpYAA or AYYAA into ApYpYAA, in the presence of $[\mathbf{E u . 1}]^{+}$resulted in measurable decreases in the intensity of the $\Delta J=2$ emission band (Figure 4a and Figure S5a), accompanied by pronounced changes in spectral form. Specifically, the intense single component $\Delta J=2$ band, characteristic of the bound diphosphorylated peptide, changed gradually to the less intense, two-component band associated with each of the other peptides. Following the emission intensity at $614 \mathrm{~nm}$ (Figure 4b), a decrease in intensity was seen upon addition of AYpYAA or AYYAA to ApYpYAA, which was approximately linear up to 
$50 \%$ conversion. The simulated dephosphorylation can also be followed ratiometrically, by monitoring the change in the intensity ratio at $614 / 599 \mathrm{~nm}$ (Figure 4c and Figure S5c). A simulation of the second dephosphorylation event, involving conversion of the mono-phosphorylated peptide AYpYAA to the non-phosphorylated peptide AYYAA, was followed in a similar manner, showing minimal changes in emission spectra (Figure S6). Based on these experiments, it was anticipated that in monitoring the enzyme-catalysed dephosphorylation of ApYpYAA, the predominant observation would be the first dephosphorylation, with minor fluctuations in emission intensity being observed for the second dephosphorylation. The byproduct of dephosphorylation is inorganic phosphate, and titration of this species into [Eu.1 $]^{+}$, in the presence of AYYAA, caused a minimal change in emission intensity and spectral form of [Eu.1 $]^{+}$(Figure S7a). Thus, the accumulation of inorganic phosphate during the enzyme reaction was not expected to interfere with the ability of $[\mathbf{E u . 1}]^{+}$to signal the dephosphorylation process. Similarly, addition of the acid phosphatase enzyme to $[\text { Eu.1 }]^{+}$also caused negligible changes in the emission spectra of [Eu.1 $]^{+}$(Figure S7b).
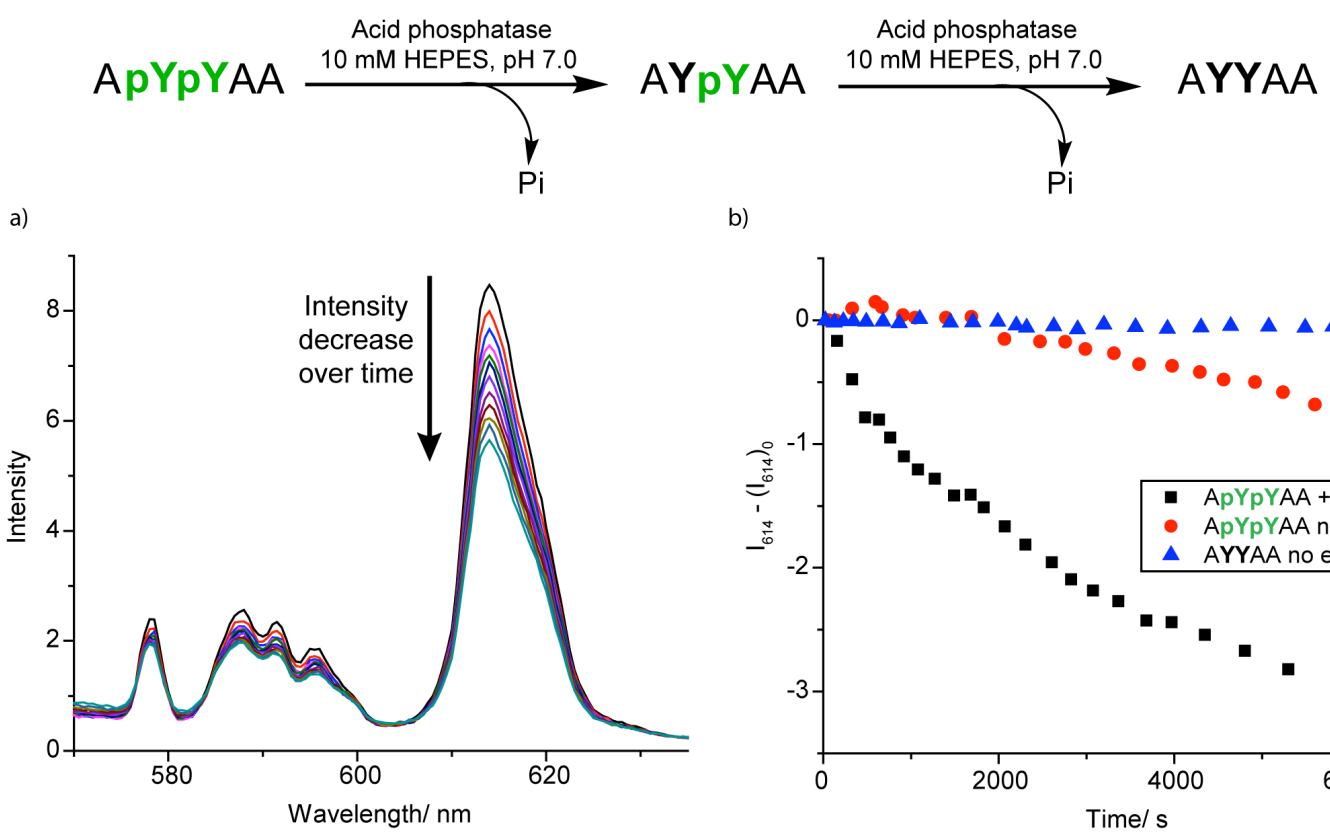

b)

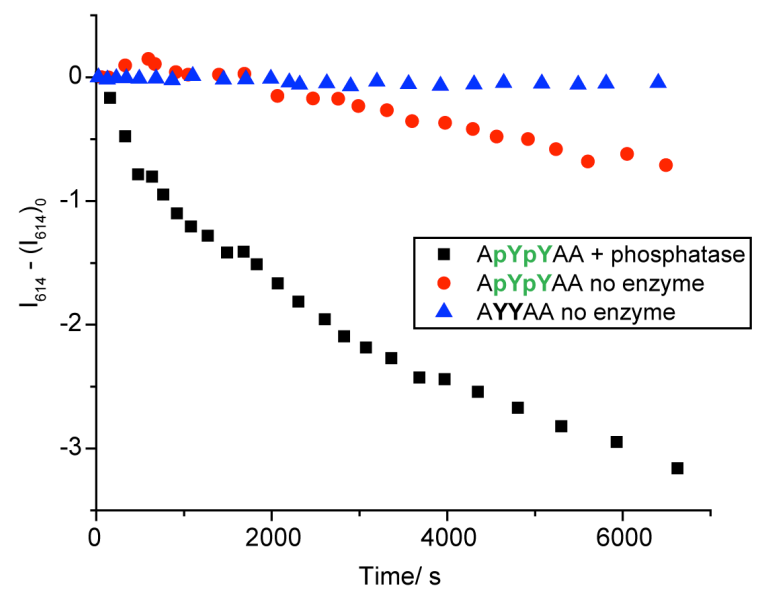


Figure 5. Luminescence monitoring of the acid phosphatase $\left(0.5\right.$ units $\left.\mathrm{mL}^{-1}\right)$ catalysed dephosphorylation of ApYpYAA ( $8 \mathrm{mM})$ using [Eu.1 $]^{+}(8 \mu \mathrm{M})$. a) Change in the emission spectrum of [Eu.1 $]^{+}(570-630 \mathrm{~nm})$; b) Change in Eu(III) emission intensity at $614 \mathrm{~nm}$ in the presence and absence of enzyme (4 mM ApYpYAA), referenced against the non-phosphorylated peptide AYYAA (4 mM). Conditions: $10 \mathrm{mM}$ HEPES buffer, $\mathrm{pH} 7.0, \lambda_{\text {exc }}=330 \mathrm{~nm}$.

Next, we used $[\mathbf{E u} .1]^{+}$to monitor the acid phosphatase catalysed dephosphorylation of ApYpYAA in real-time. Incubation of ApYpYAA (8 mM) with acid phosphatase $\left(0.5\right.$ units $\left.\mathrm{mL}^{-1}\right)$ resulted in a decrease in the intensity of the $\Delta J=2$ band of $[\mathbf{E u} .1]^{+}$over time (Figure 5a). A plot of the change in intensity at $614 \mathrm{~nm}$ as a function of time revealed a rapid decrease in the emission intensity of [Eu.1 $]^{+}$, with the rate of intensity decrease slowing over time, as expected for the dephosphorylation of the peptide (Figure $5 \mathrm{~b}$ ). In the absence of enzyme, the rate of emission intensity decrease was much slower, and can be attributed to the background hydrolysis of peptide ApYpYAA. As a control, the emission intensity of $[\mathbf{E u . 1}]^{+}$in the presence of the non-phosphorylated peptide AYYAA remained constant over the time, indicating minimal photobleaching of $[\mathbf{E u . 1}]^{+}$during the timeframe of the experiment (Figure $5 \mathrm{~b}$ ). The magnitude of the observed decrease in Eu(III) emission intensity in the presence of acid phosphatase was lower than that expected based on the titration of either AYYAA or AYpYAA into the ApYpYAA peptide. This could be due to the dephosphorylation reaction not reaching completion in the time frame studied, with some ApYpYAA remaining at the end of the experiment. This is presumably as a result of inhibition of the phosphatase enzyme by the byproduct, inorganic phosphate, a known inhibitor of the enzyme.

Having demonstrated that it was possible to monitor acid phosphatase activity, the reaction was followed at 3 different enzyme concentrations (Figure 6). Pleasingly, the rate of hydrolysis of ApYpYAA increased with increasing concentrations of 
enzyme. A plot of the initial rates of reaction revealed linear responses over the first 8 minutes (Figure 6b), which would allow for initial enzyme reaction rates to be determined. This demonstrates the utility of $[\mathbf{E u . 1}]^{+}$for monitoring the acid phosphatase catalysed hydrolysis of proximally phosphorylated tyrosine residues in real-time.

a)

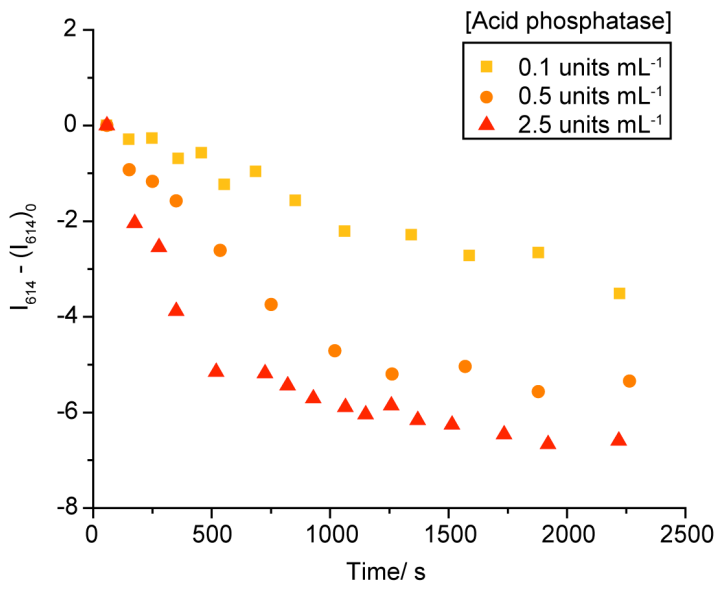

b)

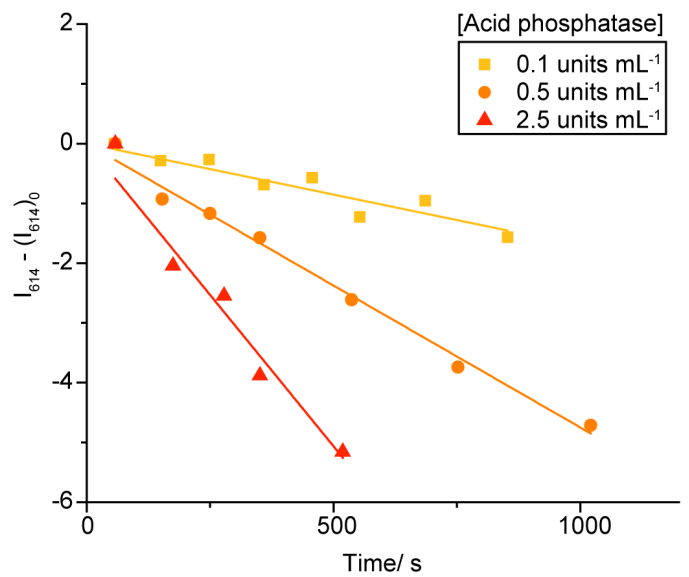

Figure 6. Monitoring of the acid phosphatase catalysed dephosphorylation of ApYpYAA (4 mM) using [Eu.1 $]^{+}(8 \mu \mathrm{M})$, using different enzyme concentrations. a) Change in $\mathrm{Eu}(\mathrm{III})$ emission intensity at $614 \mathrm{~nm}$ over time at the three different enzyme concentrations $\left(0.1,0.5\right.$ and 2.5 units $\left.\mathrm{mL}^{-1}\right)$; b) Initial rates of hydrolysis, showing linear responses over at least the first 8 minutes. Conditions: 10 mM HEPES buffer, $\mathrm{pH}$ 7.0, $\lambda_{\text {exc }}=330 \mathrm{~nm}$.

\section{Conclusion}

A stable luminescent Eu(III) complex, [Eu.1 $]^{+}$, has been shown to discriminate proximally phosphorylated tyrosine residues in peptides under neutral aqueous conditions, from the analogous mono- and non-phosphorylated peptides, as well as differentially located phosphotyrosine residues and proximally phosphorylated serine and threonine residues. The excellent discriminatory behaviour of the $\mathrm{Eu}(\mathrm{III})$ complex allowed its application in a continuous-read enzyme assay, wherein the phosphatase catalysed dephosphorylation of a peptide containing a pYpY motif was monitored in 
real-time, without the need to chemically modify the enzyme or its substrate. Future work will be directed towards the recognition of proximally phosphorylated tyrosine residues in more biologically and therapeutically important peptides and proteins, tracking their dephosphorylation by using more substrate-specific phosphatase enzymes.

\section{Acknowledgements}

This work was supported by a Wellcome Trust Seed Award (204500/Z/16/Z) and a Royal Society Research Grant. We thank Dr. David Worrall for use of fluorescence spectroscopy facilities.

\section{References}

1. Collins, B. E.; Anslyn, E. V., Chem. Eur. J., 2007, 13, 4700-4708.

2. Margulies, D.; Hamilton, A. D., Curr. Opin. Chem. Biol., 2010, 14, 705-712.

3. Kubota, R.; Hamachi, I., Chem. Soc. Rev., 2015, 44, 4454-4471.

4. Hewitt, S. H.; Wilson, A. J., Chem. Commun., 2016, 52, 9745-9756.

5. Borrebaeck, C. A. K., Immunol. Today, 2000, 21, 379-382.

6. Hunter, T., Cell, 2000, 100, 113-127.

7. Hornbeck, P. V.; Zhang, B.; Murray, B.; Kornhauser, J. M.; Latham, V.; Skrzypek, E., Nucleic Acids Res., 2015, 43, D512-D520.

8. Ojida, A.; Mito-oka, Y.; Sada, K.; Hamachi, I., J. Am. Chem. Soc., 2004, 126, 2454-2463.

9. Ojida, A.; Mito-oka, Y.; Inoue, M.-a.; Hamachi, I., J. Am. Chem. Soc., 2002, 124, 6256-6258.

10. Sakamoto, T.; Ojida, A.; Hamachi, I., Chem. Commun., 2009, 0, 141-152.

11. Kraskouskaya, D.; Bancerz, M.; Soor, H. S.; Gardiner, J. E.; Gunning, P. T., J. Am. Chem. Soc., 2014, 136, 1234-7.

12. Zhang, T.; Edwards, N. Y.; Bonizzoni, M.; Anslyn, E. V., J. Am. Chem. Soc., 2009, 131, 11976-11984.

13. Duodu, E.; Kraskouskaya, D.; Campbell, J.; Graca-Lima, G.; Gunning, P. T., Chem. Commun., 2015, 51, 6675-7.

14. Wang, X.; Yang, T.; Luo, J.; Yang, L.; Yao, C., Chem. Commun., 2015, 51, 81858188.

15. Atkinson, P.; Bretonniere, Y.; Parker, D., Chem. Commun., 2004, 0, 438-439.

16. Atkinson, P.; Murray, B. S.; Parker, D., Org. Biomol. Chem., 2006, 4, 3166-3171.

17. Ojida, A.; Inoue, M.-a.; Mito-oka, Y.; Tsutsumi, H.; Sada, K.; Hamachi, I., J. Am. Chem. Soc., 2006, 128, 2052-2058.

18. Kraskouskaya, D.; Cabral, A. D.; Fong, R.; Bancerz, M.; Toutah, K.; Rosa, D.; Gardiner, J. E.; de Araujo, E. D.; Duodu, E.; Armstrong, D.; Fek1, U.; Gunning, P. T., Analyst, 2017, 142, 2451-2459. 
19. Anai, T.; Nakata, E.; Koshi, Y.; Ojida, A.; Hamachi, I., J. Am. Chem. Soc., 2007, 129, 6232-6239.

20. Zondlo, S. C.; Gao, F.; Zondlo, N. J., J. Am. Chem. Soc., 2010, 132, 5619-5621.

21. Pazos, E.; Golicnik, M.; Mascarenas, J. L.; Eugenio Vazquez, M., Chem. Commun., 2012, 48, 9534-9536.

22. Tremblay, M. S.; Zhu, Q.; Martí, A. A.; Dyer, J.; Halim, M.; Jockusch, S.; Turro, N. J.; Sames, D., Org. Lett., 2006, 8, 2723-2726.

23. Tremblay, M. S.; Lee, M.; Sames, D., Org. Lett., 2008, 10, 5-8.

24. Gonzalez-Vera, J. A.; Bouzada, D.; Bouclier, C.; Eugenio Vazquez, M.; Morris, M. C., Chem. Commun., 2017, 53, 6109-6112.

25. Gempf, K. L.; Butler, S. J.; Funk, A. M.; Parker, D., Chem. Commun., 2013, 49, 9104-6.

26. Butler, S. J.; Parker, D., Chem. Soc. Rev., 2013, 42, 1652-66.

27. Weitz, E. A.; Chang, J. Y.; Rosenfield, A. H.; Morrow, E. A.; Pierre, V. C., Chem. Sci., 2013, 4, 4052-4060.

28. Surender, E. M.; Bradberry, S. J.; Bright, S. A.; McCoy, C. P.; Williams, D. C.; Gunnlaugsson, T., J. Am. Chem. Soc., 2017, 139, 381-388.

29. Parker, D.; Shuvaev, S.; Starck, M., Chem. Eur. J., 2017, 23, $9974-9989$.

30. Butler, S. J.; McMahon, B. K.; Pal, R.; Parker, D.; Walton, J. W., Chem. Eur. J., 2013, 19, 9511-7.

31. McMahon, B. K.; Gunnlaugsson, T., J. Am. Chem. Soc., 2012, 134, 10725-10728.

32. Palmer, A. J.; Ford, S. H.; Butler, S. J.; Hawkins, T. J.; Hussey, P. J.; Pal, R.; Walton, J. W.; Parker, D., RSC Adv., 2014, 4, 9356.

33. Butler, S. J., Chem. Commun., 2015, 51, 10879-10882.

34. Hewitt, S. H.; Parris, J.; Mailhot, R.; Butler, S. J., Chem. Commun., 2017.

35. Cabral, A. D.; Murcar-Evans, B. I.; Toutah, K.; Bancerz, M.; Rosa, D.; Yuen, K.; Radu, T. B.; Ali, M.; Penkul, A.; Kraskouskaya, D.; Gunning, P. T., Analyst, 2017.

36. Ishida, Y.; Inoue, M.-a.; Inoue, T.; Ojida, A.; Hamachi, I., Chem. Commun., 2009, 2848-2850.

37. Ojida, A.; Inoue, M.-a.; Mito-oka, Y.; Hamachi, I., J. Am. Chem. Soc., 2003, 125, 10184-10185.

38. Beeby, A.; M. Clarkson, I.; S. Dickins, R.; Faulkner, S.; Parker, D.; Royle, L.; S. de Sousa, A.; A. Gareth Williams, J.; Woods, M., J. Chem. Soc., Perkin Trans. 2, 1999, 493-504.

39. Vonci, M.; Mason, K.; Suturina, E. A.; Frawley, A. T.; Worswick, S. G.; Kuprov, I.; Parker, D.; McInnes, E. J. L.; Chilton, N. F., J. Am. Chem. Soc., 2017, 139, 14166-14172. 